Case report

\title{
Microsurgical reconstruction in giant Marjolin's ulcer of the popliteal region
}

\author{
Isabela S. Caizer ${ }^{1,2}$, Vlad A. Bloancă ${ }^{* 1,2}$, Tiberiu Bratu ${ }^{1,2}$, Zorin Crăiniceanu ${ }^{1,2}$ \\ 1 "Victor Babeș" University of Medicine and Pharmacy Timișoara, Romania; ${ }^{2}$ Department of Plastic \\ and Reconstructive Surgery, "Pius Brânzeu" Emergency County Hospital Timișoara, Romania
}

\begin{abstract}
Marjolin's ulcers are the result of malignant transformation of chronically inflamed skin or damaged skin into squamous cell carcinoma. As the most common etiologies that arise from such a transformation, it is usually a burn scar. Our study focuses on the case of a female patient admitted to our hospital with giant Marjolin's ulcer of around 20x12 cm in the popliteal fossa. Wide excision followed by free flap reconstruction was proposed. The uniqueness of the case consisted in (i) the latency period of over 42 years, (ii) the lack of metastasis and (iii) the functionality issue as a result of the affected area.
\end{abstract}

Keywords: Marjolin's ulcer; free flap; latissimus dorsi flap

\section{Introduction}

Marjolin's ulcer is an uncommon type of malignant transformation of the scarred skin that was first reported by Celsius, and later described by Marjolin in 1828 [1]. For a long time, this type of cancer was known as the burn scar neoplasm, but in the recent years, more etiologies were found especially in the case of osteomyelitis, pressure sores, chronic fistulas; essentially in all pathologies that involved chronically traumatized skin [2]. Because of the challenging diagnosis, both clinical and histologic, the incidence rate is reported at $2 \%$ [1].

Usually there is a slow growth rate, the typical latency period is 30 years, and transformations can still occur decades after the initial trauma [3].

As to the histological type, in most cases there is a well-differentiated squamous cell

Received: July 2018; Accepted after review: August 2018; Published: September 2018.

${ }^{*}$ Corresponding author: Vlad A. Bloancă, Department of Plastic and Reconstructive Surgery, "Pius Brânzeu" Emergency County Hospital Timișoara, Romania

Email: vlad.bloanca@gmail.com carcinoma, while basal cell carcinoma, sarcoma or melanoma are much lower in incidence. In around $40 \%$ of the cases the lower limb is affected, and male incidence is twice that of females [1].

Treatment is usually done through surgical intervention by wide excision of the lesion; typically with a $2 \mathrm{~cm}$ margin [1]. Chemotherapy or radiation therapy are not commonly used, except in recurrences [1].

Our case is that of a patient suffering from a giant Marjolin's ulcer measuring 20x12 cm after experiencing a burn lesion at the age of five. Biopsy revealed Marjolin's ulcer. After the patient was admitted to our ward, we removed the tumor by a wide excision leaving a defect of $24 \times 16 \mathrm{~cm}$ which was reconstructed with a latissimus dorsi flap.

\section{Case report}

A 52-year-old female presented with a giant tumor in the left popliteal fossa (about $20 \times 12 \mathrm{~cm}$ ) that has been developing over a period of five years (Figure 1a). There was no prior medical history, except to the same 
location at age five. With the exception of the tumor, her general condition was normal. The clinical examination revealed that the knee range of motion was very limited with no active or passive flexion, resulting in a stiff joint.

The lab results were normal except for a minor neutrophilia (73.2\%) and a raised fibrinogen $(549 \mathrm{mg} / \mathrm{dl})$. Upon the patient admission a sample from the tumor's surface was taken for bacterial examination. The result was positive for Proteus mirabilis. We started antibiotic therapy with Levofloxacin $5 \mathrm{mg} / \mathrm{ml}$ $100 \mathrm{ml} / 12$ hours as a first dose and continued with a single dose per day for five days.
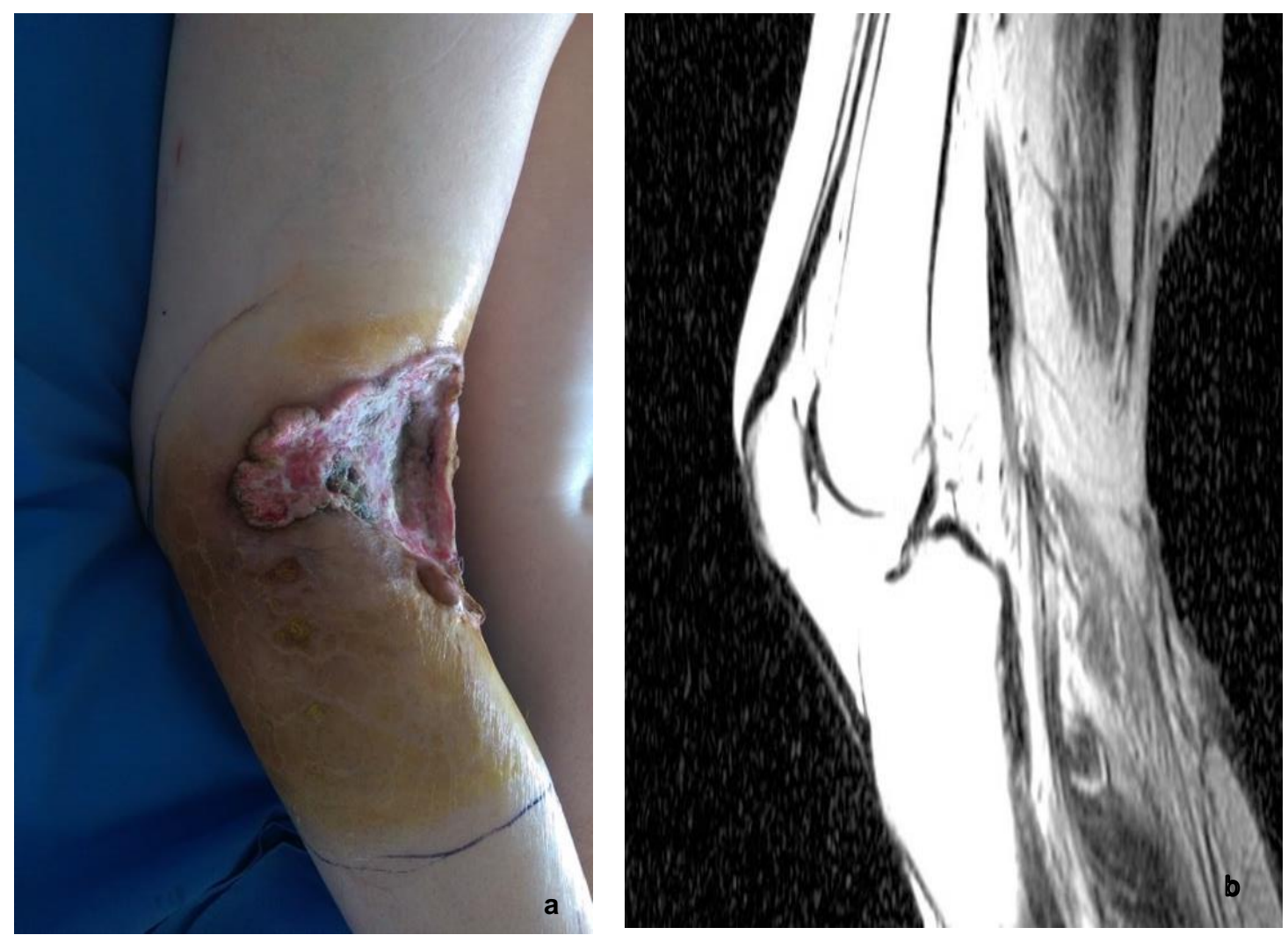

Fig. 1. Preoperative aspect of the popliteal fossa: clinic (a) and MRI (b)

After five days the antibiotic was stopped, and a new sample was taken for the bacterial examination after a rest period of 3 days. The new result came positive for Pseudomonas aeruginosa and, thus, we started with a new antibiotic treatment this time with Teicoplanin $400 \mathrm{mg}$ with a first dose of $800 \mathrm{mg}$. Following a 5-day of antibiotic and local antiseptic dressing, the lesion showed no discharge, the result of the antibiogram was negative, and the
CT scan revealed that the tumor was very well vascularized with numerous arteriovenous shunts. We've also done an MRI scan to her left knee, and a large popliteal region defect was revealed, with a liquid collection that superficially communicated with the posterior popliteal region (Figure 1b). We performed an ultrasound that revealed a normal structure inguinal lymph node (the nearest station). Sentinel lymph node assessment is not a feasible option in Marjolin's ulcers due to the small disclosure rate. The methods of choice for screening for distant metastasis were CT and MRI evaluation. 
we also performed an extemporaneous exam to confirm the clinical diagnosis of Marjolin's ulcer.

The defect in the popliteal region was a large one of $24 \times 16 \mathrm{~cm}$ (oval aspect), without intersecting the major anatomical structures present in this region (popliteal artery and vein, tibial nerve, common peroneal nerve). We performed a latissimus dorsi muscle flap to
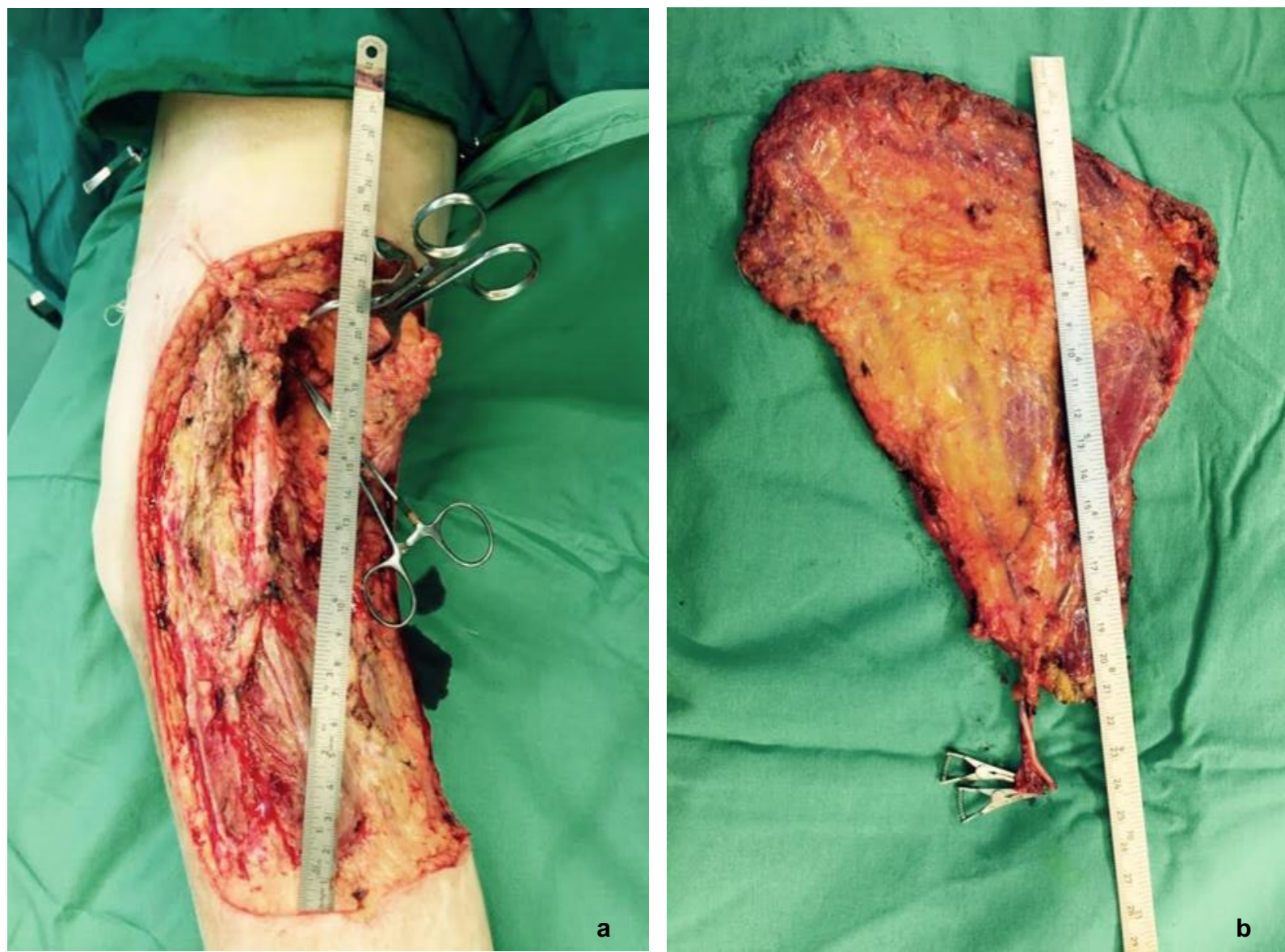

Fig. 2. An intraoperative aspect of the tissue defect (a) and the muscle flap latissimus dorsi flap (b)

The flap was covered with a split thickness skin graft once the viability of the muscle was determined (at day 5).

The result of the pathological exam was squamous cell carcinoma G1/G2 well/moderate differentiated completely resected. Due to the low aggression of the tumor and its slow evolution, this was regarded as a positive outcome. cover the defect (Figure 2b). The muscular flap was harvested from the left side and the defect was primary sutured while a microsurgical anastomosis was done: end-toside at the popliteal artery and end-to-side at the popliteal vein. The flap covered $90 \%$ of the defect, with a remnant zone in the superior region. 

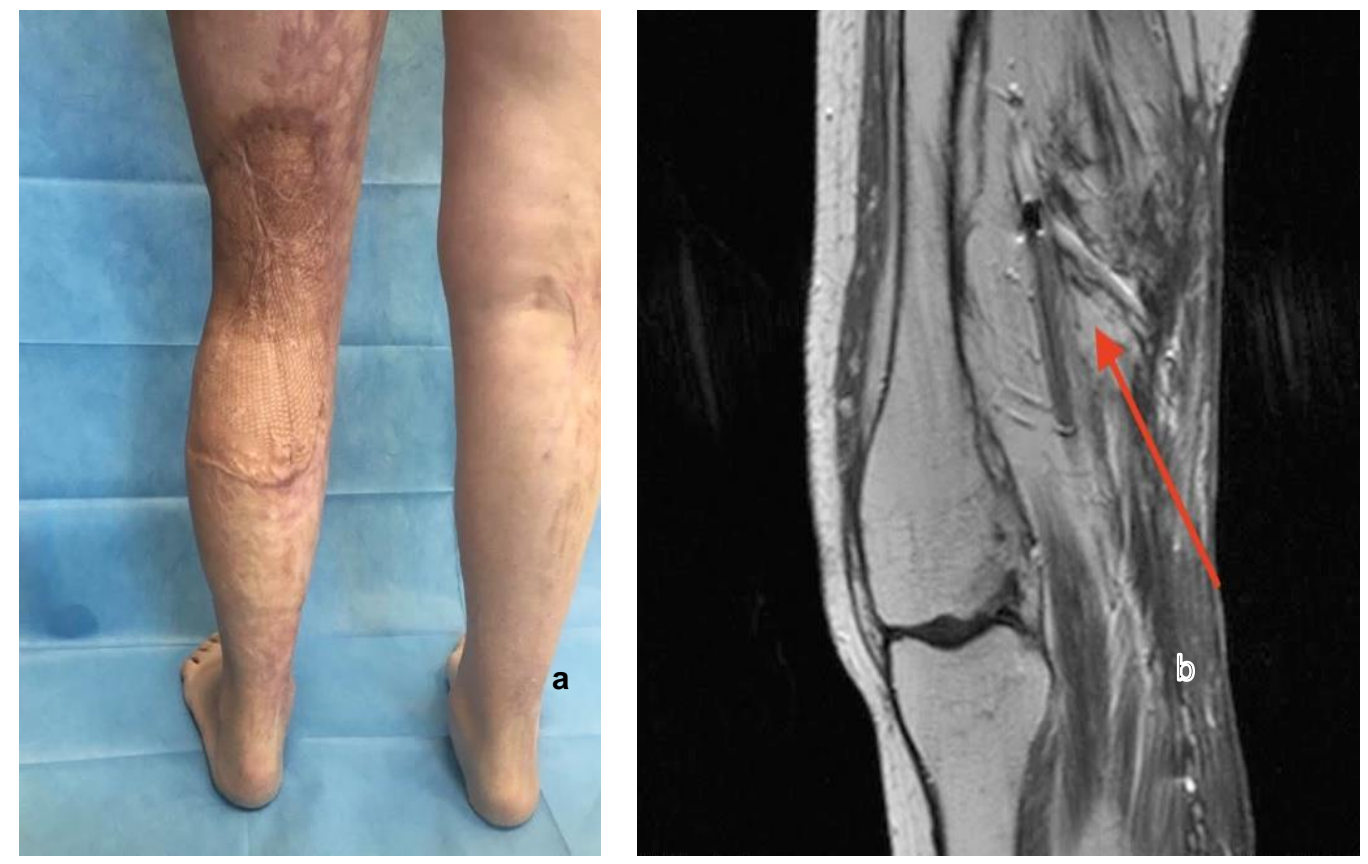

Fig. 3. Postoperative - 2 years follow-up clinic (a) and MRI aspects (b) - red arrow indicates the microsurgical anastomosis

\section{Discussion}

French surgeon Jean-Nicolas Marjolin was the one that described for the first time this kind of lesion in 1828 [4], while Dupuytren was the first to use the name malignancy when concerning Marjolin's ulcer [5].

The process from which the wound evolves into Marjolin's ulcer is uncertain. Theories acknowledge that such may be caused by inflammation for long periods of time, ultraviolet rays, or environmental interaction [6].

Marjolin's ulcer has two varieties: the acute type and the chronic one. In regard to the former, the malignant change occurs within the first 12 months from the initial injury. The latter is a process that appears slowly with an extensive latency period [7, 8]. This period between the initial trauma and the malignant result can be as long as 25-40 years [7]. Research shows that the men are more prone than women to this kind of malignant transformations [8].

The treatment for this variety of cancer should be multidisciplinary, and it should be done in oncological units. Depending on the case, treatment for these lesions should include surgery, but also chemotherapy or radiotherapy like in selected cases of recurrences [10]. From a surgical standpoint, Marjolin's ulcer treatment should include first of all a wide excision with circumferential assessment of the margins. If the lymph nodes results are positive, lymphadenectomy is recommended. Only as last resort, if the tumor is too large the amputation should be considered $[8,9]$.

Skin grafting after a wide excision is chosen in some Marjolin's ulcer cases. The margins which should be at least $2 \mathrm{~cm}[9,10]$, as these are considered microscopically safe margins [11] It is important to obtain these safety margins because of the aggressiveness of such tumors.

The particularity of this case consists in the reconstruction of the defect by latissimus dorsi free flap transfer for coverage due to the nature and the dimensions of the defect in a region of high demanding functionality as the knee joint. An important step of the treatment is the way of covering this kind of defect. In our case, we opted for a muscular free flap because it offered more protection for sensible anatomical structure of the affected region, while also covered a large concave defect. Grafting was not a viable option for the primary closure, due to (i) the anatomical structures in 
that region, (ii) the poor aesthetic result, (iii) the risk of knee contracture, (iv) the safe margins $(2 \mathrm{~cm})$ required, and $(v)$ the aim of achieving a very low risk of recurrence.

In the case of our patient, the malignant transformation occurred after 42 years, a latency period much longer than foreseen in prior research on the matter.

The patient evolution was good. Two years later there were no secondary determinations of the tumor and no lymph nodes involved, so the vital prognostic is good. The patient recovered the full extension of the knee, with a great functionality result. The aesthetic result was only a satisfactory one, due to the skin graft scars.

Another key aspect of our case is the microorganisms that the ulcerated tumor had. The first was Proteus mirabilis, a Gramnegative facultative anaerobic found in soil and water, while the second pathogen found was Pseudomonas aeruginosa that is a Gramnegative, multidrug-resistant pathogen associated with hospital infections. The germ is considered opportunistic and is usually found in immunocompromised patients [12].

This rare case showed the great challenges in treatment when dealing with such massive tumors in a high functionality region like the knee articulation.

\section{References}

1. Jayasree K, Quadri SS, Umamaheswar Rao BN, Kumar DK. Marjolin's ulcer - dual lymph node metastasis with cystic degeneration: a rare presentation. Int Surg J 2014; 1(1):56-59.

2. Ochenduszkiewicz $U$, Matkowski R, Szynglarewicz B, Kornafel J. Marjolin's ulcer: malignant neoplasm arising in scars. Rep Pract Oncol Radiother 2006; 11(3):135-138.

3. Pekarek B, Buck S, Osher L. A comprehensive review on Marjolin's ulcers: diagnosis and treatment. J Am Coll Clin Wound Spec 2011; 3(3):60-64.

4. Marjolin NJ. Ulcère. Dictionnaire de medicine 1828; $21: 31$

5. Treves N, Pack GT. The development of cancer in burn scars. Surg Gynecol Obstet 1930; 51:749-782.

6. Nthumba PM: Marjolin's ulcers: theories, prognostic factors and their peculiarities in spina bifida patients. World J Surg Oncol 2010; 8:108.

\section{Conclusions}

Marjolin's ulcer is a particular squamous cell carcinoma, an aggressive variety that is found after a long period of latency in chronic wounds, especially in burn scars.

This rare condition is rather complicated due to the affected area, and surgical treatment becomes challenging especially the reconstructive one during which the functionality of the patient must be kept.

The primary reconstruction raised oncological and bacteriological risks due to tissue defect characteristic, vessels and exposed nerves, but, fortunately it ended in a good functional and acceptable cosmetic result.

When dealing with Marjolin's ulcer, the latency period can be misleading since it may occur even after long periods of time than those generally anticipated. Despite the normal course of the tumor, it is essential to offer our patients a good outcome, both functional and aesthetic.

\section{Competing interests}

The authors have no conflict of interests to declare.

\section{Patient consent}

Written informed consent was obtained from the patient's legal guardians for publication of this case.

7. Wysocki WM, Komorowski A, Wojewoda T, et al. Two different cases of Marjolin's ulcer and recommendations for practice. TOSJ 2010; 2:83-85.

8. Davalos BA, Cortes-Flores AO, BanderaDelago A. Malignant neoplasm in burn scar: Marjolin's ulcer: report of two cases and review of the literature. Cir Cir 2008; 76:329-331.

9. Malheiro E, Pinto A, Choupina M. Marjolin's ulcer of the scalp: case report and literature review. Ann Burns Fire Disasters 2001; 14:3941.

10. Ochenduszkiewicz $U$, Matkowski R, Szynglarewicz B, et al. Marjolin's ulcer: malignant neoplasm arising in scars. Rep Pract Oncol Radiother 2006; 11(3):135-138.

11. Opara KO, Otene IC. Majorlin's Ulcers: A Review. TNHJ 2011; 11(4):107-111.

12. Sadikot RT, Blackwell TS, Christman JW, Prince AS. Pathogen-host interactions in Pseudomonas aeruginosa pneumonia. $A m \mathrm{~J}$ Respir Crit Care Med 2005; 171(11):1209-1223 